\title{
Psychological Cognition of Women of Childbearing Age on the Use of Cosmetics During Pregnancy
}

\author{
Jing Huo, Hongling Fan, Hui Liu, Chunjing Zhao, Xiuli Hou* \\ Department of Dermatology, Dezhou People's Hospital, Dezhou 253000, Shandong Province, China \\ *Corresponding author: Xiuli Hou, dermhou@163.com
}

\begin{abstract}
Objective: To investigate the psychological cognition of women of childbearing age on the use of cosmetics during pregnancy, so as to provide guidance for women of childbearing age to correctly use cosmetics during pregnancy. Methods: A total of 180 women of childbearing age who underwent gynecological examination in Dezhou People's Hospital from October 2020 to October 2021 were selected as the research subjects. Questionnaires were used to investigate the psychological cognition of women of childbearing age on the use of cosmetics during pregnancy. Results: Among the 180 women of childbearing age, 48 (26.67\%) felt that cosmetics cannot be used during pregnancy, 60 (33.33\%) felt that it did not matter, and $72(40.00 \%)$ agreed that cosmetics can be used during pregnancy. In addition, urban women, highly educated women, unmarried women, and enterprise employees agreed with the use of special cosmetics for pregnant women or the selective use of cosmetics during pregnancy. Conclusion: Most women of child-bearing age felt that cosmetics can be used during pregnancy; this cognition is related to their education level, occupation, and registered residence.
\end{abstract}

Keywords: Women of childbearing age; Cosmetics; Pregnancy; Psychological cognition

Publication date: November 2021; Online publication: November 30, 2021

\section{Introduction}

Young women of childbearing age have an increasing demand for diversified skin products. With the development and evolvement of cosmetics, the evaluation and research on the effect of cosmetics are more in-depth, especially for women of childbearing age with reproductive needs. Recently, due to the rapid development of the national economy, people's quality of life has significantly improved, and the public demand for cosmetics has doubled. Dermatology, pharmacology, physiology, and embryology have begun to penetrate into cosmetic science. Cosmetics with high safety profile and certain physiological effects are gradually being accepted by consumers ${ }^{[1]}$. In order to explore the cognition of women of childbearing age on the use of cosmetics during pregnancy, a questionnaire survey was carried out on 180 women of childbearing age who underwent obstetrics and gynecology examination in Dezhou People's Hospital from October 2020 to October 2021. The results from the survey were then analyzed.

\section{Materials and methods}

\subsection{General data}

A total of 180 women of childbearing age who underwent obstetrics and gynecology examination in Dezhou People's Hospital from October 2020 to October 2021 were selected as research subjects. Their cognition on the use of cosmetics during pregnancy was investigated using questionnaires. All the women had signed the informed consent in advance. Besides, the intelligence and physical health of all the subjects were normal and would not have affected the experimental results. 


\subsection{Methods}

Questionnaires were distributed to 180 women of childbearing age to investigate their cognition on the use of cosmetics during pregnancy. The main contents of the questionnaire are as follows:

(1) epidemiological investigation - the collection of basic information of women of childbearing age, including age, education, occupation, registered residence, and working environment;

(2) investigation on the cognition of using cosmetics during pregnancy in order to determine whether women of childbearing age use cosmetics as well as their cognition and understanding on the use of cosmetics during pregnancy.

\subsection{Statistical analysis}

SPSS 25.0 software was used to analyze the data. The data were presented as $\bar{x} \pm \mathrm{s}$ or in percentage. The differences between two groups were compared by t-test, while those among three groups or above were contrasted using chi-square test. $P<0.05$ indicates a significant difference.

\section{Results}

\subsection{Basic information of women of childbearing age}

A total of 180 women of childbearing age were investigated, of which $120(66.67 \%)$ of them were of age 20 to 30, $60(33.33 \%)$ were in the age group of 31 to 40, and there were none above 40 years old. There were 150 married women (83.33\%) and 30 unmarried women (16.67\%). As for education level, there were 40 women who received education up to junior middle school $(22.22 \%)$, 58 women with high school or technical secondary school education (32.22\%), 42 women with college education (23.33\%), 30 women with undergraduate education (16.67\%), and 10 women with education level up to graduate or a higher level (5.56\%). Besides, 93 of them were employees (51.67\%), 34 farmers (18.89\%), 26 self-employed workers $(14.44 \%)$, and 27 of them being unemployed (15.00\%). A hundred and eight women were living in cities and 72 were living in rural areas.

\subsection{Use of cosmetics by women of childbearing age and their cognition on the use of cosmetics during pregnancy}

After investigation, among the 180 women of childbearing age, $80(44.44 \%)$ of them often use cosmetics, $68(37.78 \%)$ occasionally use cosmetics, and $32(17.78 \%)$ women do not use cosmetics. The survey on whether cosmetics can be used during pregnancy found that 48 women $(26.67 \%)$ felt that cosmetics cannot be used, 60 (33.33\%) felt that it did not matter, and 72 women (40.00\%) agreed that cosmetics can be used (Table 1). It was notable that highly educated women, city registered residence women, and enterprise employees had higher recognition of cosmetics during pregnancy.

Table 1. Cognition of women of childbearing age on the use of cosmetics during pregnancy

\begin{tabular}{lccc}
\hline & Cannot be used & Does not matter & Can be used \\
\hline Age & & & \\
$20 \sim 30$ & 16 & 55 & 49 \\
$31 \sim 40$ & 32 & 5 & 23 \\
\hline Marital status & & & 15 \\
Unmarried & 5 & 50 & 57 \\
Married & 43 & \multicolumn{2}{c}{ Continued on the next page }
\end{tabular}




\begin{tabular}{lccc}
\hline & Cannot be used & Does not matter & Can be used \\
\hline Educational background & & & \\
Junior middle school & 18 & 13 & 9 \\
High school or technical secondary school & 10 & 32 & 16 \\
Junior college & 10 & 22 & 10 \\
Undergraduate & 6 & 10 & 14 \\
Graduate and above & 4 & 2 & 4 \\
\hline Occupation & & & 54 \\
Staffs or workers of enterprise & 14 & 25 & 11 \\
Farmers & 18 & 19 & 4 \\
Self-employed & 3 & 11 & 3 \\
Unemployed & 13 & & 49 \\
\hline Household type & & 28 & 23 \\
Urban & 31 & 32 & \\
Country & 17 & & \\
\hline
\end{tabular}

\section{Discussion}

Cosmetics have become a necessity in the daily life of a modern woman. With the gradual increase in incidence of abnormal menstruation among modern women, spontaneous abortion rate, birth defects, infertility, and other phenomena, more emphasis has been on the subject of healthy fertility among women of childbearing age. The use cosmetics among women of childbearing age has also become a research hotspot ${ }^{[2]}$. However, there are only a few reports on the psychological perceptions of women of childbearing age about the use of cosmetics during pregnancy.

In this study, a questionnaire survey was carried out on 180 women of childbearing age. The results showed that most women of childbearing age agreed that cosmetics can be used during pregnancy; however, most of them felt that special skin care products for pregnant women should be used, otherwise there would be adverse effects on the fetus. In addition, the psychological cognition of women of childbearing age was found to be closely related to their age, educational background, living environment, and working conditions. Generally speaking, highly educated women living in cities and office workers are more likely to use appropriate cosmetics during pregnancy, which do not have adverse effects on both mothers and infants; instead, they foster the feeling of happiness among mothers and are conducive to fetal development. In conclusion, the cognition of women of childbearing age on the use of cosmetics during pregnancy has a certain bias, which is closely related to their age, living environment, educational background, and working conditions.

\section{Disclosure statement}

The authors declare that there is no conflict of interest.

\section{References}

[1] Chen Z, Zhang Q, Gan Z, 2004, To Explore the Effect of Cosmetics on the Content of Lead and Cadmium in Hair of Pregnant Women and Their Newborns. Journal of Luzhou Medical College, 27: 316-318. 
[2] Li F, Zhang Y, Pang J, et al., 2014, Health Related Toxicity of Cosmetic Ingredients to Women of Childbearing Age and Current Situation of Domestic Safety Management. Capital Public Health, 8: 127-130. 